\title{
Physical properties of the nuclear region in Seyfert galaxies derived from EVN observations
}

\author{
M. Giroletti ${ }^{* a}$, F. Panessa ${ }^{b}$, M. Orienti ${ }^{a, c}$, A. Doi ${ }^{d}$ \\ ${ }^{a}$ INAF - Istituto di Radioastronomia, via Gobetti 101, 40129 Bologna, Italy \\ ${ }^{b}$ INAF/IAPS via del Fosso del Cavaliere 100, 00133 Roma, Italy \\ ${ }^{c}$ Dipartimento di Astronomia, Università di Bologna, via Ranzani 1, 40127 Bologna, Italy \\ ${ }^{d}$ Institute of Space and Astronautical Science, JAXA, 3-1-1 Yoshinodai, Sagamihara, Kanagawa \\ 229-8510, Japan \\ E-mail: girolettieira.inaf.it
}

\begin{abstract}
We report on sensitive dual-frequency (1.7 and $5 \mathrm{GHz}$ ) European VLBI Network observations of the central region of nine Seyfert galaxies. These sources are among the faintest and least luminous members of a complete sample of nearby $(d<22 \mathrm{Mpc})$ low luminosity AGNs. We detect radio emission on milliarcsecond scale in the nuclei of 4 galaxies, while for the other five sources we set an upper limit of $<100 \mu \mathrm{Jy}$. In three sources, namely NGC 3227, NGC 3982, and NGC 4138, radio emission is detected at both 1.7 and $5 \mathrm{GHz}$ and it is resolved in two or more components. We describe the structural and spectral properties of these features; we find that in each of these three nuclei there is one component with high brightness temperature (typically $\left.T_{B}>10^{7} .5 \mathrm{~K}\right)$ and flat/intermediate spectral index $\left(0.3<\alpha<0.6, S(v) \sim v^{-\alpha}\right)$, accompanied by secondary steep spectrum extended components. In these cases, non-thermal emission from jets or outflows is thus the most natural explanation. A faint feature is detected in NGC 4477 at $5 \mathrm{GHz}$; keeping in mind the modest significance of this detection $(\sim 5 \sigma)$, we propose the hot corona as the origin of non-thermal emission, on the basis of the unrealistic magnetic field values required by synchrotron self-absorption. Finally, the five non-detected nuclei remain elusive and further observations on intermediate scales will be necessary to investigate their nature.
\end{abstract}

11th European VLBI Network Symposium \& Users Meeting,

October 9-12, 2012

Bordeaux, France

* Speaker. 


\section{Background}

We are studying a sample of 28 local Seyfert galaxies to understand the physical properties of their nuclear regions. X-ray observations reveal nuclear activity at low luminosity levels in most of them [2], but what about the radio emission? Ho\&Ulvestad (2001) [5] revealed flux density at the milliJansky level in $80 \%$ of them, using the VLA at 1.4 and $5 \mathrm{GHz}$. Various authors exploited the high resolution of VLBI to clarify the nature of the cores of the brightest sources. Possible interpretations include thermal free-free emission from an X-ray heated corona or two-sided jet-like structures with low speeds, indicating non-relativistic jet motion, possibly due to thermal plasma as in NGC 4151.

\section{EVN enters the game: old and new observations}

For the faintest sources in the sample, the extraordinary sensitivity of the European VLBI Network is a key resource. We reported in Giroletti \& Panessa (2009) the results of dual-frequency EVN observations for 5 objects, resulting in clear detections for 4 of them, at least at one frequency [4]. Motivated by the success of the previous experiment, we observed eight more targets with the same setup, including also the Arecibo radio telescope for sources with suitable declination. We clearly revealed three sources at two frequencies, plus one source at $5 \mathrm{GHz}$ only. Low significance components are found also in other sources but it is hard to claim real detections in these cases. A detailed discussion of these observation and of the statistical analysis of the whole sample is provided in dedicated papers $[1,7]$.

\section{Notes on single sources}

NGC 3227 - This Sy1.5 core shows a compact (1.2 mas), flat spectrum ( $\alpha \sim 0.6)$ component, with brightness temperature $\sim 10^{7.5} \mathrm{~K}$, so presumably of non thermal nature. At about $12 \mathrm{pc}$ in position angle (PA) $170^{\circ}$ and $9 \mathrm{pc}$ in $\mathrm{PA}-45^{\circ}$, we find two more extended, steep spectrum regions. This VLBI structure connects nicely to the larger scale emission observed in literature MERLIN images [6].

NGC 3982 - The nuclear region of this Sy1.9 is resolved in two components, one with more compact structure ( 0.9 mas) and flatter spectrum $(\alpha \sim 0.4)$; the $T_{B}$ is $\sim 10^{8.5} \mathrm{~K}$, suggestive of non thermal emission. The other component is $8.5 \mathrm{pc}$ away and it is more resolved and with steeper spectrum.

NGC 4138 - NGC4138 is Sy1.9 galaxy. Our EVN observations reveal a main component, detected at the two frequencies, with size $\sim 1.4$ mas, flat spectral index $(\alpha \sim 0.3)$ and $T_{B} \sim 10^{9.1} \mathrm{~K}$. At $1.6 \mathrm{GHz}$, a second component is detected $\sim 50$ mas westward ( $3.5 \mathrm{pc}$ ), more extended.

\section{Physical properties}

All the sources detected at both frequencies, i.e. NGC3227, NGC3982 and NGC4138, present one component with high brightness temperature $\left(\log T_{B}>7.5\right)$ and flat spectral index $(0.3 \leq \alpha \leq$ 0.6 ), which we ascribe to non-thermal emission from the immediate vicinity of the central black 
Table 1: Physical quantities at $1.7 \mathrm{GHz}$. Col. 3: brightness temperature; col. 4: monochromatic luminosity; col. 5: volume; col. 6: minimum energy density; col. 7: equipartition magnetic field.

\begin{tabular}{llccccc}
\hline Source & Component & $\begin{array}{c}\log T_{B} \\
(\mathrm{~K})\end{array}$ & $\begin{array}{c}\log L \\
\left(\mathrm{~W} \mathrm{~Hz}^{-1}\right)\end{array}$ & $\begin{array}{c}\log V \\
\left(\mathrm{~cm}^{-3}\right)\end{array}$ & $\begin{array}{c}\log U_{\min } \\
\left(\mathrm{erg} \mathrm{cm}^{-3}\right)\end{array}$ & $\begin{array}{c}B_{\mathrm{eq}} \\
(\mathrm{mG})\end{array}$ \\
\hline NGC 3227 & $\mathrm{C}$ & 7.5 & 19.8 & 54.1 & -5.76 & 4.3 \\
& $\mathrm{~S}$ & 6.5 & 20.5 & 56.9 & -6.90 & 1.2 \\
& $\mathrm{~N}$ & 6.1 & 19.7 & 55.9 & -6.83 & 1.3 \\
$\mathrm{NGC} 3982$ & $\mathrm{C}$ & 9.1 & 19.8 & 52.0 & -4.53 & 17.8 \\
& $\mathrm{~S}$ & 7.2 & 20.0 & 54.9 & -6.14 & 2.8 \\
$\mathrm{NGC} 41388$ & $\mathrm{C}$ & 8.5 & 19.4 & 52.1 & -4.86 & 12.2 \\
& $\mathrm{~W}$ & 6.6 & 18.8 & 54.8 & -6.73 & 1.4 \\
\hline
\end{tabular}

Table 2: Physical quantities at $5 \mathrm{GHz}$. Columns as in Table 1.

\begin{tabular}{llccccc}
\hline Source & Component & $\begin{array}{c}\log T_{B} \\
(\mathrm{~K})\end{array}$ & $\begin{array}{c}\log L \\
\left(\mathrm{~W} \mathrm{~Hz}^{-1}\right)\end{array}$ & $\begin{array}{c}\log V \\
\left(\mathrm{~cm}^{-3}\right)\end{array}$ & $\begin{array}{c}\log U_{\min } \\
\left(\mathrm{erg} \mathrm{cm}^{-3}\right)\end{array}$ & $\begin{array}{c}B_{\mathrm{eq}} \\
(\mathrm{mG})\end{array}$ \\
\hline NGC 3227 & $\mathrm{C}$ & 7.5 & 19.5 & 52.4 & -4.96 & 10.9 \\
& $\mathrm{~S} 1$ & 5.4 & 19.1 & 54.9 & -6.61 & 1.6 \\
& $\mathrm{~S} 2$ & 5.8 & 19.1 & 54.4 & -6.31 & 2.3 \\
$\mathrm{NGC} 3982$ & $\mathrm{C}$ & 7.6 & 19.7 & 52.5 & -4.93 & 11.2 \\
& $\mathrm{~S}$ & 5.7 & 19.3 & 54.7 & -6.37 & 2.1 \\
$\mathrm{NGC} 4138$ & $\mathrm{C}$ & 7.8 & 19.2 & 51.4 & -4.52 & 18.1 \\
$\mathrm{NGC} 4477$ & $\mathrm{C}$ & 6.5 & 18.7 & 52.7 & -5.59 & 5.2 \\
\hline
\end{tabular}

hole; steep spectrum extended components are also detected within some tens of parsecs from the core, suggesting the presence of jets or outflows on parsec scales. In Tables 1 and 2, we report physical parameters for the various components estimated under the assumption of minimum energy. The parameters we derive are reasonable; e.g. the equipartition magnetic field is of a few mG. On the other hand, in NGC 4477, detected only at $5 \mathrm{GHz}$, the brightness temperature is lower, and the physical parameters are at odd with a synchrotron self-absorption (SSA) scenario, mainly because of the too high magnetic field required; a thermal free-free origin for its radio emission seems more viable, similar to NGC 1068 [3]. The undetected sources remain a mystery; since they have weak but compact cores in VLA images, e-MERLIN observations would be ideal to investigate their nature.

\section{Acknowledgments}

The European VLBI Network is a joint facility of European, Chinese, South African and other radio astronomy institutes funded by their national research councils. This effort/activity is supported by the European Community Framework Programme 7, Advanced Radio Astronomy in Europe, grant agreement No. 227290. We acknowledge a contribution from the Italian Foreign Affair Minister under the bilateral scientific collaboration between Italy and Japan.

\section{References}

[1] Bontempi, P., Giroletti, M., Panessa, F., Orienti, M., \& Doi, A. Physical properties of the nuclear region in Seyfert galaxies derived from observations with the European VLBI Network, MNRAS,426, 588, [arXiv:1208.0230] 
[2] Cappi, M., Panessa, F., Bassani, L., et al. X-ray spectral survey with XMM-Newton of a complete sample of nearby Seyfert galaxies, $A \& A, 446,459$, [arXiv:astro-ph/0509584]

[3] Gallimore, J. F., Baum, S. A., \& O'Dea, C. P. The Parsec-Scale Radio Structure of NGC 1068 and the Nature of the Nuclear Radio Source, ApJ,613, 794, [arXiv:astro-ph/0406062]

[4] Giroletti, M., \& Panessa, F. The Faintest Seyfert Radio Cores Revealed by VLBI, ApJ,706, L260, [arXiv:0910.5821]

[5] Ho, L. C., \& Ulvestad, J. S. Radio Continuum Survey of an Optically Selected Sample of Nearby Seyfert Galaxies, ApJS,133, 77, [arXiv:astro-ph/0102506]

[6] Mundell, C. G., Holloway, A. J., Pedlar, A., Meaburn, J., Kukula, M. J., \& Axon, D. J. Anisotropic radio and optical emission in the Seyfert nucleus of NGC 3227, MNRAS,275, 67

[7] Panessa, F., \& Giroletti, M. Sub-parsec radio cores in nearby Seyfert galaxies, MNRAS,432, 1138, [arXiv:1304.0794] 